\title{
Front Matter: Volume 9449
}

, "Front Matter: Volume 9449," Proc. SPIE 9449, The International Conference on Photonics and Optical Engineering (icPOE 2014), 944901 (20 February 2015); doi: 10.1117/12.2186572

Event: The International Conference on Photonics and Optical Engineering SPIE. China 


\title{
PROCEEDINGS OF SPIE
}

\section{The International Conference on Photonics and Optical Engineering (icPOE 2014)}

\author{
Ailing Tian \\ Anand Asundi \\ Weiguo Liu \\ Chunmin Zhang \\ Editors
}

13-15 October 2014

Xi'an, China

\section{Organized by}

Xi'an Technological University (China) •Xi' an Jiaotong University (China) • Xi' an Institute of Optics and Precision Mechanics (China) • Xi'an Institute of Applied Optics (China) • Nanyang Technological University (Singapore) • Centre for Optical and Laser Engineering, Nanyang Technical University

(Singapore)

Sponsored by

Shaanxi Optical Society (China) • Shaanxi Provincial Physical Society (China) • Optics and Photonics Society of Singapore (Singapore)

Technical Co-sponsor

SPIE

Cooperating Organization

Council Units of Shaanxi Optical Society (China)

Supported by

Ministry of Science and Technology of China $\bullet$ Chinese Academy of Sciences $\bullet$ National Natural Science Foundation of China

Volume 9449

Part One of Two Parts 
The papers included in this volume were part of the technical conference cited on the cover and title page. Papers were selected and subject to review by the editors and conference program committee. Some conference presentations may not be available for publication. The papers published in these proceedings reflect the work and thoughts of the authors and are published herein as submitted. The publisher is not responsible for the validity of the information or for any outcomes resulting from reliance thereon.

Please use the following format to cite material from this book:

Author(s), "Title of Paper," in The International Conference on Photonics and Optical Engineering (iCPOE 2014), edited by Ailing Tian, Anand Asundi, Weiguo Liu, Chunmin Zhang, Proceedings of SPIE Vol. 9449 (SPIE, Bellingham, WA, 2015) Article CID Number.

ISSN: 0277-786X

ISBN: 9781628415650

\section{Published by}

\section{SPIE}

P.O. Box 10, Bellingham, Washington 98227-0010 USA

Telephone +1 3606763290 (Pacific Time) · Fax +1 3606471445

SPIE.org

Copyright @ 2015, Society of Photo-Optical Instrumentation Engineers.

Copying of material in this book for internal or personal use, or for the internal or personal use of specific clients, beyond the fair use provisions granted by the U.S. Copyright Law is authorized by SPIE subject to payment of copying fees. The Transactional Reporting Service base fee for this volume is $\$ 18.00$ per article (or portion thereof), which should be paid directly to the Copyright Clearance Center (CCC), 222 Rosewood Drive, Danvers, MA 01923. Payment may also be made electronically through CCC Online at copyright.com. Other copying for republication, resale, advertising or promotion, or any form of systematic or multiple reproduction of any material in this book is prohibited except with permission in writing from the publisher. The CCC fee code is 0277-786X/15/\$18.00.

Printed in the United States of America.

Publication of record for individual papers is online in the SPIE Digital Library.

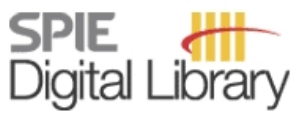

SPIEDigitalLibrary.org

Paper Numbering: Proceedings of SPIE follow an e-First publication model, with papers published first online and then in print. Papers are published as they are submitted and meet publication criteria. A unique citation identifier (CID) number is assigned to each article at the time of the first publication. Utilization of CIDs allows articles to be fully citable as soon as they are published online, and connects the same identifier to all online, print, and electronic versions of the publication. SPIE uses a six-digit CID article numbering system in which:

- The first four digits correspond to the SPIE volume number.

- The last two digits indicate publication order within the volume using a Base 36 numbering

system employing both numerals and letters. These two-number sets start with 00, 01, 02, 03, 04, $05,06,07,08,09,0 A, 0 B \ldots$. OZ, followed by 10-1Z, 20-2Z, etc.

The CID Number appears on each page of the manuscript. The complete citation is used on the first page, and an abbreviated version on subsequent pages. 


\title{
Contents
}

\section{Part One}

\author{
xi Authors \\ xv Conference Committees \\ xvii Introduction
}

INVITED PAPER SESSION

944902 Design, fabrication, and evaluation of diffractive optical elements for generation of focused ring patterns (Invited Paper) [9449-221]

944903 Large mode area waveguides by volume ultrafast laser photoinscription (Invited Paper) [9449-282]

944904 Riesz transforms in statistical signal processing and their applications to speckle metrology: a review (Invited Paper) [9449-239]

944905 Phase unwrapping algorithm based on double-frequency fringe projection and fringe background (Invited Paper) [9449-140]

\section{SPACE OPTICS}

944906 A fast ATP method based on integral chopper [9449-30]

944907 Theoretic and numerical analysis of diamagnetic levitation and its experimental verification [9449-45]

944908 Infrared image non-rigid registration based on regional information entropy demons algorithm [9449-61]

944909 Correction of group refraction index based on pulse trains interference [9449-70]

9449 OA Simulation of the atmospheric $\mathrm{CO}_{2}$ measurements at Fuling with the inversion model results [9449-95]

9449 OB The optical scattering property: spatial and angle variability in Northern South China Sea [9449-133]

9449 OC Non-resolved space object characterization with space-based brightness data [9449-163]

9449 OD Swapping transmit of quantum entanglement information in the process of unresonant interaction by intensity-dependent coupling [9449-168] 
9449 OE Decoding algorithms and spatial resolution Monte Carlo simulation of cross strip anode for UV astronomy [9449-169]

9449 OF MTF online compensation in space optical remote sensing camera [9449-170]

9449 OG A method for generating multichannel entangled photon pairs [9449-193]

$9449 \mathrm{OH} \quad$ Comparison between the ESFT method and LBL method of $\mathrm{CO}_{2}$ retrieval for high-resolution satellite [9449-213]

9449 Ol Study on polarization properties of particle's scattered light [9449-224]

9449 0J The design of space optical communications terminal with high efficient [9449-267]

9449 OK Phase-space analysis of transport of intensity equation under partially coherent illumination [9449-291]

$9449 \mathrm{OL}$ On two heuristic viewpoints concerning the study of light [9449-309]

\section{SPECTRAL IMAGING TECHNIQUE}

9449 OM Concept of an autostereoscopic system containing 29 million of stereoscopic image pairs [9449-5]

$9449 \mathrm{ON} \quad$ Study on thermal effect model of laser irradiating moving and rotating target [9449-12]

944900 The phase error reduction using window functions in digital holography [9449-27]

9449 OP High-precision method of deleting infrared energy of background for flying target [9449-53]

$9449 \mathrm{OQ}$ Color image encryption using iterative phase retrieve process in quaternion Fourier transform domain [9449-58]

9449 OR Disparity search range estimation based on TOF-stereo fusion [9449-91]

9449 OS Generation of two-wavelength infrared optical signals based on coupled parametric sixwave mixings [9449-113]

9449 OT The characteristic analysis of spectral image for cabbage leaves damaged by diamondback moth pests [9449-151]

9449 OU Absolute spectral responsivity of silicon trap detector based on absolute cryogenic radiometer [9449-171]

9449 OV Research on hyperspectral dynamic infrared scene simulation technology [9449-172]

9449 OW Study on Raman spectra of synthetic celluloses [9449-185]

9449 0X Spectrum recovery method analysis on nonuniform sampling interference data [9449-227] 
9449 OY Research on error compensation method for spectral reconstruction in color reproduction [9449-228]

$9449 \mathrm{OZ}$ Evaluation of tea quality by two-dimensional fluorescence correlation spectroscopy [9449-257]

944910 Photocatalytic inactivation effect of $\mathrm{HMME}-\mathrm{TiO}_{2}$ nanocomposites on SCC cells under visible light [9449-272]

944911 Deep ultra-violet beam profile measurement of KrF laser using laser induced fluorescence [9449-313]

\section{PHOTOELECTRIC MATERIAL AND PHOTOELECTRIC DEVICES}

944912 Research on the mathematical and simulated models of photovoltaic cells for laser power beaming in space [9449-10]

944913 Upconversion photoluminescence and EXAFS of $\mathrm{Er}^{3+}$ and $\mathrm{Ho}^{3+}$ codoped with $\mathrm{Li}^{+}$ion [9449-36]

944914 Total internal reflection diffraction grating in conical mounting and its application in planar display [9449-48]

944915 Design and simulation of e-calendar system circuits [9449-50]

944916 Humidity sensing of microfiber Bragg grating [9449-55]

944917 A kind of transmission-type lens using in x-ray band [9449-67]

944918 Ultrafast thermal excitation behaviors of Au films irradiated by polarization-shaped femtosecond laser [9449-71]

944919 Research of the relations between mono layer SiNx optical thin film processing techniques and laser induced damage properties prepared by PECVD technology [9449-101]

$94491 \mathrm{~A} \quad$ Optimal optical communication terminal structure for maximizing the link budget [9449-103]

9449 1B Surface conduction electron emission source enhanced by back gate electrode [9449-138]

9449 1C An improved scheme that the random matrix transformation used for BB84 protocol [9449-139]

9449 1D Readout circuit design of the retina-like CMOS image sensor [9449-157]

$94491 \mathrm{E} \quad$ Estimating the complex index of refraction based on the rough surface polarization degree [9449-178]

9449 IF Study of the polarized characteristics of laser echo for rough surface based on PG model [9449-182] 
9449 1G Compact diode-pumped nanosecond $\mathrm{Nd}: \mathrm{YVO}_{4}$ grazing-incidence slab amplifier [9449-190]

$94491 \mathrm{H} \quad$ The building and simulation of color analytical model based on prism dispersion [9449-203]

$944911 \quad$ A new designed impedance matching circuit used for gated framing camera [9449-217]

9449 iJ A new method to increase the anti-laser damage ability of DLC films [9449-219]

$94491 \mathrm{~K}$ Transmission loss in x-ray framing cameras [9449-220]

$94491 \mathrm{~L} \quad$ Photonic crystal based anti-reflection structure for GaN/InGaN heterojunction solar cells [9449-243]

9449 1M Study of optical stability for vertical high-power white light-emitting diodes [9449-246]

$94491 \mathrm{~N} \quad$ Study on the temperature characteristics of LPFG magnetic field sensor based on magnetic fluid [9449-248]

944910 Research on influence of environmental conditions on measurement performance of magnetic field sensor based on differential group delay in FBG [9449-249]

9449 IP Analysis on the temperature property of sensors based on symmetrical metal-cladding optical waveguide [9449-250]

$94491 Q \quad$ Optimization and analysis of near-infrared InGaAs detectors [9449-284]

9449 IR Design and analysis of AlGalnP-based light emitting diodes with $\mathrm{SiO}_{2}$ current blocking layer [9449-289]

9449 is Design of low-dispersion output coupler for Cr:LiSAF lasers [9449-293]

9449 1T A novel fabrication of fiber Bragg grating in hollow-core fiber with HPDLCs [9449-304]

\section{Part Two}

\section{OPTICAL DESIGN AND MANUFACTURING TECHNOLOGY}

$94491 \mathrm{U}$ Design and simulation of flat-top microstructure fiber [9449-2]

$94491 \mathrm{~V}$ The pump parameters optimization in LDA pumped solid-state laser [9449-8]

$94491 \mathrm{~W}$ The investigation of automatic exposure under extreme light [9449-33]

$94491 \mathrm{X} \quad$ High-sensitivity Mach-Zehnder interferometric refractive index sensor based on two different ultra-abrupt tapers [9449-34]

9449 IY Optical fiber core diameter mismatched in-fiber Mach-Zehnder interferometer for strain sensing [9449-43] 
944912 Error analysis and design of the optical system in airborne remote sensing measurement [9449-54]

944920 Research and design of portable photoelectric rotary table data-acquisition and analysis system [9449-57]

944921 Design and implementation of photoelectric rotary table data acquisition and analysis system host computer software based on VC++ and MFC [9449-59]

944922 Dynamic optical aberration correction with adaptive coded apertures techniques in conformal imaging [9449-79]

944923 A design of camera simulator for photoelectric image acquisition system [9449-81]

944924 Design of infrared signal processing system based on heterogeneous MPSoC [9449-84]

944925 Research on process technology of off-axis ellipsoid aspheric mirror [9449-100]

944926 Research on ultrafast laser polishing monocrystalline-silicon [9449-102]

944927 Design and analysis of the satellite laser communications network [9449-116]

944928 Research on the error model of airborne celestial/inertial integrated navigation system [9449-135]

944929 A two-step high-precision distortion correction algorithm of wide-angle lens [9449-145]

9449 2A Optical design of electro-optical UAV-based reconnaissance and surveillance system with shared aperture [9449-149]

9449 2B Dual FOV infrared lens design with the laser common aperture optics [9449-153]

9449 2C Design of multi-spectral images real-time segmentation system [9449-158]

9449 2D Continuous zooming optical design with a high resolution and a large-zoom ratios used for precision strike TV camera [9449-159]

$94492 \mathrm{E}$ Design of an off-axis reflecting dual-band common optical path optical system [9449-176]

$94492 \mathrm{~F}$ Design and simulation for a testing system of color space resolution [9449-209]

$94492 \mathrm{G}$ Research of wind loading on optical window for airborne optoelectronic equipment based on CFD [9449-210]

$94492 \mathrm{H} \quad$ A new autofocus method based on angular spectrum method in digital holography [9449-214]

$944921 \quad$ Role of the incident beam on Super-RENS disc system with high-numerical aperture [9449-222]

9449 2J The design of laser scanning galvanometer system [9449-253] 
$94492 \mathrm{~K}$ The status and trends of microscopic indirect imaging system with super resolution [9449-280]

9449 2L Research on solar-blind UV optical imaging system [9449-300]

$94492 \mathrm{M}$ The study of optical properties of InGaN-based solar cell with blue emission wavelength [9449-301]

$94492 \mathrm{~N}$ Optical system design for wide-angle airborne mapping camera with diffractive optical element [9449-310]

\section{OPTICAL MEASURING AND TESTING}

944920 A circuit used for peak power detecting of the laser pulse [9449-69]

$94492 \mathrm{P} \quad$ Colorimetric pyrometry system design and calibration experiment [9449-73]

$94492 \mathrm{~A}$ A new surface temperature measurement using infrared detector [9449-76]

$94492 \mathrm{R} \quad$ High-precision positioning system of four-quadrant detector based on the database query [9449-92]

$94492 \mathrm{~S}$ Real-fringe DASH interferometer for upper atmospheric wind and temperature observation: concept and simulation [9449-109]

9449 2T Automatic stitching interferometry for large plano optics [9449-1 19]

$94492 \mathrm{U}$ On-line measurement of diameter of hot-rolled steel tube [9449-126]

$94492 \mathrm{~V}$ Experimental research of error restraint for dynamic interferometer in optical testing [9449-134]

$94492 \mathrm{~W}$ Optical measurement of interface movements of liquid metal excited by a pneumatic shaker [9449-154]

$94492 X \quad$ The influence of the tip shape on the STM scans [9449-167]

$94492 Y$ Measurement of InGaAs single photon detector quantum efficiency at 1550nm [9449-173]

944922 Numerical calculation of aerosol transmittance on transmission route [9449-212]

944930 Measurement of Rayleigh-Brillouin spectra in gas flows [9449-218]

944931 Study on phase demodulation technique with fast and high accuracy for interference fringe pattern [9449-225]

944932 Interferogram interpolation method research on TSMFTIS based on kernel regression with relative deviation [9449-226]

944933 Absolute testing of surface based on sub-aperture stitching interferometry [9449-236] 
944934 Study of ultrafast laser polishing technology [9449-238]

944935 High-precision radius automatic measurement using laser differential confocal technology [9449-242]

944936 Equipment for measuring of transmission of infrared optic materials in high temperature [9449-244]

944937 Research and theoretical analysis of new fiber Bragg grating sensor demodulation system [9449-247]

944938 An optical fiber multiplexing interferometric system for measuring remote and high precision step height [9449-252]

944939 Design and analysis of range finder for medium distance [9449-258]

9449 3A Geometric measurement of moving object based on visual detecting-learning mechanism [9449-271]

9449 3B Study on short distance laser ranging system [9449-274]

$94493 \mathrm{C}$ Influence of shearing displacement error study on two-dimensional lateral shearing interferometry [9449-275]

9449 3D Precision 3D surface measurement of step-structures using mode-locked femtosecond pulses [9449-288]

$94493 \mathrm{E} \quad$ Real-time and non-destructive method for coaxial cylinder core diameter measurement [9449-290]

9449 3F A method of multi-view intraoral 3D measurement [9449-302]

$94493 \mathrm{G}$ Enhancing the photon number resolving capability of multi-pixel photon counter by waveform integral of the avalanche pulses [9449-307]

$94493 \mathrm{H}$ Performances of a solid streak camera based on conventional CCD with nanosecond time resolution [9449-311]

$944931 \quad$ Application of laser-induced plasma spectroscopy for nuclear material analysis and detection: a comprehensive review [9449-312]

MICRO-NANO MANUFACTURING AND TESTING

9449 3J Research on detection technology of microstructure of optical surface based on total integrated scattering method [9449-52]

9449 3K Design and analysis of 2D photonic crystals for nano-structured solar cells [9449-77]

9449 3L Application of finite difference to study morphology evolution during etching the optical surface with subsurface damage [9449-87] 
$94493 \mathrm{M} \quad$ Advances in simulation study on organic small molecular solar cells [9449-90]

$94493 \mathrm{~N} \quad$ Fabrication of 3D solenoid microcoils in silica glass by femtosecond laser wet etch and microsolidics [9449-137]

944930 Microstructure measurement of digital holography [9449-229]

9449 3P Study of high aspect ratio silicon etching based on ICP [9449-254]

$94493 Q \quad$ The chemisorption of different elements on the Si (001) surface [9449-255]

$94493 R \quad$ Lithography process of micropore array pattern in Si microchannel plates [9449-281]

$944935 \quad$ Passive micromixers with dual helical channels [9449-292]

9449 3T Study of PIN electrical modulation structure based on SiGe-OI material [9449-294]

$94493 \mathrm{U} \quad$ Study on laser intensity modulation by spherical inclusion in silica subsurface [9449-295]

9449 3V Research on deep silicon etching for microchannel plates [9449-296] 


\section{Authors}

Numbers in the index correspond to the last two digits of the six-digit citation identifier (CID) article numbering system used in Proceedings of SPIE. The first four digits reflect the volume number. Base 36 numbering is employed for the last two digits and indicates the order of articles within the volume. Numbers start with 00, 01, 02, 03, 04, 05, 06, 07, 08, 09, OA, OB...0Z, followed by 10-1Z, 20-2Z, etc.

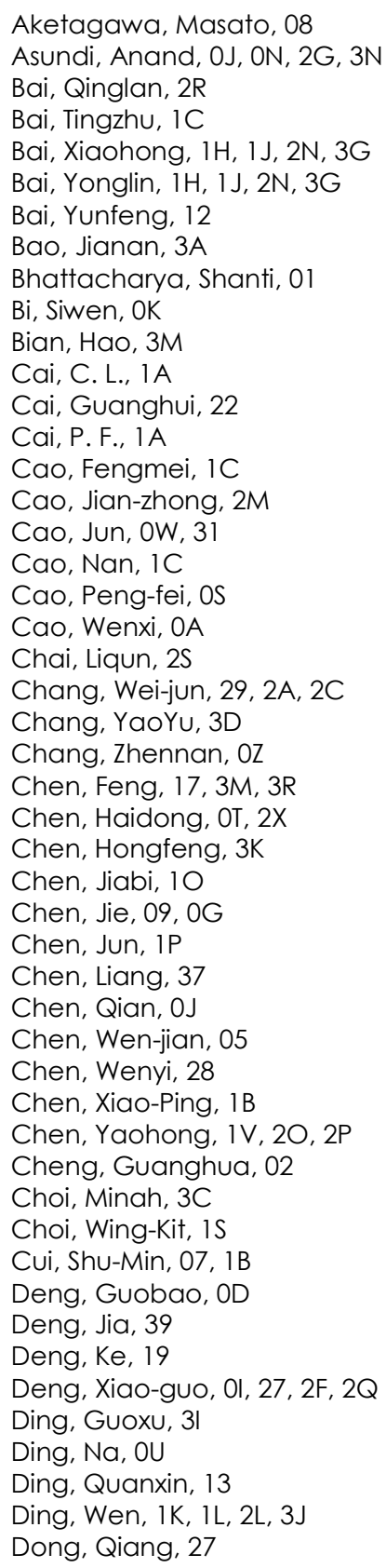

Dong, Yongjiang, OY

Du, Guangqing, 17, 3M

Du, Jiaqiang, 3U

Duan, Chengpeng, 2O, 2P

Duan, Kuaikuai, OP

Duan, Yuan-yuan, 05

Duan, Zhiyong, 06

Fan, Linlin, $3 Q$

Fan, Zheyuan, 20

Fei, Jia-qi, 2M

Fei, Xiaoyun, 2R

Feng, Jie, OS

Feng, Song, 3S

Feng, Yutao, 2R

Fu, Haiwei, 15, 1X

Gao, Bo, 25

Gao, Hong, $1 X$

Gao, Jiaobo, oU

Gao, Kun, 2J

Gao, Yong, 35

Gao, Zhi-Xing, 10, 3H

Gao, Zhi-Yuan, 1Q

Gou, Yongsheng, 1H, 1 J, 2N, $3 G$

Grasnick, Armin, OL

Guo, Jun-chao, 05

Guo, Maofeng, $1 \mathrm{~K}$

Guo, Maofeng, 1L, 2L, 3J

Guo, Wenge, 3L

Guo, Yongkui, 34

Han, Jun, 25, 33, 3Q

Han, Junfeng, 1Z, 20

Han, Kunye, 29

Han, Lixuan, 3H

Han, Yameng, OE, 2B

Han, Yaofeng, $1 \mathrm{U}$

Hang, Lingxia, 18

Hanson, Steen G., 03

$\mathrm{He}$, Yuhang, $2 \mathrm{~S}$

He, Yulan, 2D

He, Yulu, $\mathrm{OZ}$

Hong, Hua, 1B

Hou, Xun, 1K, 1L, 2L, 3J, 3M

Hsu, Chih-Hao, is

$\mathrm{Hu}, \mathrm{Bin}, 21$

$\mathrm{Hu}$, Jiang, 30, $3 \mathrm{U}$

$\mathrm{Hu}$, Jinbing, 10

$\mathrm{Hu}$, Shuai, $3 \mathrm{O}$

$\mathrm{Hu}$, Wenhua, $2 \mathrm{H}$

$\mathrm{Hu}, \mathrm{Yu}, \mathrm{OU}$ 
Huang, Fabin, 18

Huang, Fengzhen, OW, 31

Huang, Jian, 19

Huang, Jijang, $1 \mathrm{~V}$

Huang, Yaping, 1K, 3J

Hui, Lei, 38

Hui, Yongling, IF

Jang, Heesuk, 3C

Jia, Xin, 32

Jia, Zhenan, 15

Jia, Zonghe, 24

Jiang, Bo, 0 l

Jiang, Dagang, 19

Jiang, Hongwei, 34

Jiang, Hongzhi, 3E

Jiang, Menghua, IF

Jiang, Qiang, 10

Jiang, Ren-ke, 35

Jiang, $X \mathrm{U}, 2 \mathrm{E}$

Jiang, Yong, 3T

Jiang, Zhuangde, 3K

Jin, Jing, $1 \mathrm{~J}$

Jin, Linlin, 26

Kang, Wenli, 29, 2D

Kim, Seung-Woo, 3C

Kim, Young-Jin, 3C

Lee, Keunwoo, 3C

Lei, Hong, $1 \mathrm{~F}$

Leng, Han-bing, $2 M, 2 P$

Li, Bin, 2T

Li, Cai, OA

Li, Gang, Ol, $2 F$

Li, Gaoping, 2 Z

Li, Hong-ning, OS

Li, Huidong, $1 X$

Li, Jicheng, 3A, 3N

$\mathrm{Li}$, Jingzhen, OW, 31

Li, Linjun, 18

Li, Lipin, 15

Li, Ming, 3L

Li, Peng, 18

Li, Qiang, IF

Li, Qiang, 1K, 1L, 2L, 3J

Li, Qiang, 2S

Li, Tao, $2 Z$

Li, Wei, OF

Li, Wenqiang, 13

Li, XiaoGang, 25, 33

Li, Xiaojiang, OM, 11

Li, Xiao-li, 16

Li, Xiaoming, $1 G$

Li, Xue, IP

Li, Yan, 16

Li, Yan, 21

Li, Yan, $2 Z$

Li, Yanfen, 09, 0G

$\mathrm{Li}$, Yanyang, 3M, 3R

Li, Yaocheng, 1M, 1N, 36

Li, Yuan, 2D

Liang, Binming, 10
Lianga, H. F., IA

Liao, Chunyan, IR

Liao, Jiawen, IV

Liao, Ningfang, $\mathrm{OX}$

Lin, Li-bo, OS

Lin, Pin-Chung, 3D

Liu, Ang, $2 S$

Liv, Baiyu, 1H, 1 J, 2N, $3 \mathrm{G}$

Liu, Bing-cai, 30, 3B, 3N

Liu, Changming, OT, $2 X$

Liu, Dongdong, 09, 0G

Liu, Dongmei, 24

Liu, Guoyan, 2J

Liv, Huan, 3Q

Liu, Keyin, 3M, 3R

Liu, Li-jun, 14

Liv, Lina, 3F

Liu, Qiang, 26

Liv, Qing, 1D, 1E

Liu, Qinpeng, 1X

Liv, Rongxia, $1 \mathrm{~T}$

Liv, Shuo, $1 \mathrm{~L}$

Liu, Shuo, $2 \mathrm{~L}$

Liv, Shuo, 1K, 3J

Liu, Ting, 30

Liu, Wei, 2O, 2P

Liu, Weiguo, 30

Liu, Wen, 22

Liu, Xin, 02

Liu, Yinggang, 15

Liu, Yujie, 13

Liu, Yulong, OT

Lu, Chaoliang, 07

Lu, Fei, $2 Z$

Lu, Hao, OY

Lu, Xiaofei, 00

Lu, Yu, 17

Luan, Ya-dong, $2 \mathrm{~A}$

Luo, Xuexue, 10

LV, Chao, IY

LV, Jiabing, IP

LV, Peijun, 3E

Ma, Caihong, 10, 3H

$\mathrm{Ma}, \mathrm{Ke}, 24$

Ma, Li, IQ

Ma, Li-Hua, 07, 1B

Ma, Ning, 03

Ma, Sen, 37

Ma, Shi-wei, 05

Ma, Wentao, 3L

Mei, Jiansheng, $0 Z$

Men, Shouqiang, $2 \mathrm{~V}$

Meng, Sen, 3L

Meng, Xiangwei, 3M, 3R

$\mathrm{Ni}$, Guoqiang, 2J

Nie, Jing, $3 \mathrm{U}$

Niu, Hai-Jun, $2 M$

Niu, Wei, $2 \mathrm{H}$

Ou, Yan, 17

Pan, Yongqiang, $\mathrm{OH}$ 
Park, Jiyong, 3C

Peng, Yu, OF

Qian, Fengchen, 26

Qiang, Hua, 2C

Qiao, Xueguang, 15, $1 \mathrm{X}$

Qin, Feng, OS

Qin, Junjun, 1H, 1J, 1R, 2N, 3G

Qiu, Rong, 3T

Qu, Weijuan, 2G

Qu, Youshan, OE, 2B

Ren, Long, 20

Ren, Pei-an, 26

Rong, Piao, 09, 0G

Ruan, Ningjuan, 2K

Shan, Chao, 3M, 3R

Shao, Min, 15, 1X

Shen, Guang-Di, 1Q

Shen, Xiufeng, IW

Shi, Lei, 1B

Shi, Qingping, OF

Shi, Xueshun, OT, 2X

Song, Miao, $2 \mathrm{~W}$

Song, Panting, $\mathrm{OZ}$

Song, Shengyu, 1C

Song, Yinglin, 12

Stoian, Razvan, 02

Su, Junhong, 11

Su, Xilin, 1K, 3J

Su, Xiu-qin, $2 Q$

Su, Yufeng, 06

Sui, Liansheng, OP

Sun, Anxin, $1 Y$

Sun, Cheng-ming, $\mathrm{OB}$

Sun, Dandan, OU

Sun, Kefeng, OU

Sun, Xiaoling, 21

Sun, Yunchun, 3E

Sun, Zhe, IF

Takeda, Mitsuo, 03

Tang, Hengjing, IP

Tang, Xinyi, 23

Tang, Xiuzhang, $3 \mathrm{H}$

Tian, Ailing, $\mathrm{OH}, 2 \mathrm{~T}, 30,3 \mathrm{~B}, 3 \mathrm{~N}$

Tian, Bingxin, 2E

Tian, Mengqi, 2W

Tian, Runni, 3T

Tian, Yujun, $\mathrm{OH}$

Tong, Na, OR, OV

Vijayakumar, A., 01

Wang, Baohua, 2K

Wang, Bo, 1H, 1J, 2N, 3G

Wang, Chunhui, $\mathrm{OH}, 3 \mathrm{~N}$

Wang, Chunyang, 31

Wang, Da-sen, 30

Wang, Dingyi, 09

Wang, Dingyi, OG

Wang, Hairong, 3K

Wang, Hai-tong, 1M, 1N, 36

Wang, Hong, 39

Wang, Hongjun, $\mathrm{OH}, 38,3 \mathrm{~B}, 3 \mathrm{~N}$
Wang, Jiajia, OX

Wang, Jiangteng, $2 \mathrm{~L}$

Wang, Jin, 1K, 3J

Wang, Jing, $O Z$

Wang, Ju-Xia, OC

Wang, Jun, OU

Wang, Junbo, 3T

Wang, Kexuan, 11

Wang, Lei, 35

Wang, Ling, 2C

Wang, Lon A., 1S, 3D

Wang, Mengmeng, $\mathrm{OZ}$

Wang, Mingxiang, IP

Wang, Nan, 11

Wang, Nanxi, 2D

Wang, Ping, 36

Wang, Qiang, 3l

Wang, Qingsong, 1P

Wang, Shuai, 2L

Wang, Sumei, IW

Wang, Wei, 03

Wang, Yan, $2 Y$

Wang, Yawei, $1 G$

Wang, Yawei, $3 Q$

Wang, Yuanyuan, 2K

Wang, Yunzhi, 37

Wang, Yuxiao, 12

Wang, Zefeng, 1V, 2P

Wang, Zelin, $\mathrm{OZ}$

Wang, Zhaomin, 2G

Wang, Zhen, 3E

Wei, Dong, 08

Wei, Shaopeng, $0 Q$

Wen, Yongfu, 2G

Wu, Chengjing, IT

Wu, Deng-shan, 2M

Wu, Junfang, 3P

Wu, Lei, $2 Z$

Wu, Qingrong, 07

Wu, Shenjiang, 11

Wu, Wenli, OU

Wu, Wenmin, OX

Wu, Xiaoyan, $2 \mathrm{U}$

Wu, Xin, $2 \mathrm{U}$

Wu, Yanmin, 17

Xia, Deyang, 1K, 1L, 2L, 3J

Xiao, Lihui, 3K

Xiao, Yang, 34

Xie, Fang, 37

Xie, Hui, 25, 33

Xie, Junhu, OU

Xie, Qingsheng, 1V , 2O, 2P

Xie, Weihao, 21

Xie, Weimin, 32

Xie, Xiao-fang, $2 Y$

Xin, Zhou, 35

Xing, Tingwen, 32

Xiong, Jianwen, $\mathrm{OZ}$

$X \cup$, Chen, $1 Q$

$\mathrm{Xu}$, Fuchao, 32 
$X U$, Jingwen, $2 \mathrm{~V}$

$\mathrm{XU}$, Kaiyuan, $2 \mathrm{~S}$

$\mathrm{Xu}, \mathrm{Ke}, 29$

$X \cup$, Le, $1 \mathrm{~W}$

$X U, L U, 28$

XU, Peng, 1J, 2N, 3G

$\mathrm{Xu}$, Rongguo, 35

$\mathrm{Xu}$, Xiangyan, 16

$\mathrm{XU}$, Yan, $1 \mathrm{~T}$

$X U, Y$ Yang, $3 E$

$X U$, Zhantang, OA

$X U, Z$ Zhi-Yan, 1B

Xue, Bing, OR

Yan, A-qi, 2M

Yan, Chunsheng, OY

Yan, Facai, ON

Yan, Hao, ON

Yan, Huimin, $0 Q$

Yan, Peipei, Ol

Yan, $\mathrm{XU}, 1 \mathrm{X}$

Yang, Dawei, 1Z, 20

Yang, Di, 1D, 1E

Yang, Fang, 2G

Yang, Hongru, 1U, $2 Z$

Yang, Hui, 28

Yang, Jiamiao, 34

Yang, Lechen, OT

Yang, Lei, IV

Yang, Qing, 17, 3M, 3R

Yang, Shu-ming, OS

Yang, Xiaoxu, 0I, 1Z, 20, 27, 2F

Yang, Xiufang, 1Z, 20

Yang, Yewei, OM, 11

Yang, Yuansheng, 11

Yang, Yuezhong, OA

Yang, Zijian, 29

Yao, Cuping, $0 Z$

Ye, Zhitong, 06

Yi, Bo, 2O, 2P

Yin, Wanhong, 35

Ying, Che, $1 Y$

Yu, Ming, 07

Yu, Xun, 1G, 2E

Yu, Yingjie, $2 U$

Yuan, Liang, $2 Z$

Yun, Feng, 1K, 1L, 2L, 3J

Zeng, Change, 1D, $1 \mathrm{E}$

Zhai, Bo, OE, 2B

Zhai, Xuejun, OR

Zhan, Chunlian, $2 Z$

Zhan, Yonghong, 1D, 1E

Zhang, Binglong, 2

Zhang, Bo, 2A, 2C

Zhang, Chunmin, 09, 0G

Zhang, Chunwei, 04

Zhang, Cong, 3A, 3B

Zhang, Donglai, OM, 11

Zhang, Guoqing, 3F

Zhang, Haifeng, IV

Zhang, Hao, 23
Zhang, Jian, $2 M$

Zhang, Kaisheng, IV

Zhang, Linna, $2 \mathrm{U}$

Zhang, Linzhao, 1K, 1L, 3J

Zhang, Peng, 19

Zhang, Pengbin, 21

Zhang, Ruofan, $1 \mathrm{U}$

Zhang, Shun, 03

Zhang, Xiaoqiang, $2 U$

Zhang, Xin, 22, 2Q

Zhang, Xiuda, $O Q$

Zhang, Xuan, 3L

Zhang, Xuan-zhi, 29, 2A

Zhang, Ye, 1K, 1L, 2L, 3J

Zhang, Yixin, OV

Zhang, Yuangeng, 2l

Zhang, Yunshan, IX

Zhang, Zhaohui, IV

Zhang, Zhenxi

Zhang, Zhi, IV

Zhao, Fei, OB

Zhao, Hong, 04

Zhao, Huijie, 3E

Zhao, Huiying, $2 T$

Zhao, Kun, OT, 2X

Zhao, Liang, 3

Zhao, Na, IX

Zhao, Weiqian, 34

Zhao, Xin, 1M, IN

Zhao, Xue, 15

Zhao, Yulong, 3R

Zheng, Min, 1K, 1L, 3J

Zheng, Xiao-qiang, 27, 2F, 2Q

Zhong, Xiaoming, 2K

Zhou, Bin, 2 l

Zhou, Guangxi, 2W

Zhou, Jiang, $\mathrm{OE}, 2 \mathrm{~B}$

Zhou, Jiawu, 13

Zhou, Jun, 2V

Zhou, Long, 12

Zhou, Shun, 30, 3U

Zhou, Zhi-liang, OB

Zhu, Bingli, 1H, 1 J, 2N, $3 \mathrm{G}$

Zhu, Changjun, OR, OV, 3F

Zhu, PengFei, 25, 33

Zhu, Xiangping, OD

Zhu, Xiuhong, $1 R$

Zhu, Xue-liang, 2T, 30, 3N

Zhu, Yufeng, 30, 3U

Zuo, Chao, oJ 


\section{Conference Committees}

Honorary Chairs

Xun Hou, Chinese Academy of Sciences (China)

Zhuangde Jiang, Xi' an Jiaotong University (China)

General Chairs

Weiguo Liu, Xi'an Technological University (China)

Chunmin Zhang, Xi'an Jiaotong University (China)

Wei Zhao, Xi'an Institute of Optics and Precision Mechanics (China)

Dongxu Cui, Xi'an Institute of Applied Optics (China)

Anand Asundi, Nanyang Technological University (Singapore)

Academic Committee

Jintao Bai, Northwest Polytechnical University (China)

Feng Chen, Xi'an Jiaotong University (China)

Qihuang Gong, Chinese Academy of Science (China)

Junjie Guo, Xi'an Jiaotong University (China)

G. G. Shepherd, York University (Canada)

Jun Han, Xi'an Technological University (China)

Xun Hou, Chinese Academy of Science (China)

Ming Ji, Xi' an Institute of Applied Optics (China)

Zhuangde Jiang, Xi'an Jiaotong University (China)

Bing Li, Xi'an Jiaotong University (China)

Fuli Li, Xi' an Jiaotong University (China)

Qinxue Li, Xi' an Institute of Applied Optics (China)

Wenqing Liu, Chinese Academy of Engineering (China)

Ting Mei, Xi' an Electronics Science and Technology University (China)

Takeda Mistuo, Utsunomiya University (Japan)

Yongqiang Pan, Xi'an Technological University (China)

S. Hanson, Technical University of Denmark (Denmark)

Junhong Su, Xi' an Technological University (China)

Qingxi Tong, Chinese Academy of Science (China)

W. Osten, Stuttgart Universität (Germany)

Dingyi Wang, Xi'an Jiaotong University (China)

Wei Wang, Heriot-Watt University (United Kingdom)

Zhaoxin Wu, Xi'an Jiaotong University (China)

Hongru Yang, Xi' an Institute of Applied Optics (China)

Jianfeng Yang, Xi' an Institute of Optics and Precision Mechanics

(China)

Tingwu Yang, Chinese Flight Test Establishment (China) 
Baoli Yao, Xi'an Institute of Optics and Precision Mechanics (China)

Xun Yu, Xi'an Technological University (China)

Xiaocong Yuan, Shenzhen University (China)

Feng Yun, Xi'an Jiaotong University (China)

Xiaodong Zeng, Xi' an Electronics Science and Technology University

(China)

Zhenxi Zhang, Xi'an Jiaotong University (China)

Huiying Zhao, Xi'an Jiaotong University (China)

Jianlin Zhao, Northwest Technical University (China)

Hairong Zheng, Shaanxi Normal University (China)

Organizing Committee

Anand Asundi, Nanyang Technological University (Singapore)

Chen Feng, Xi'an Jiaotong University (China)

Dongxu Cui, Xi'an Institute of Applied Optics (China)

Haibo Ge, Xi'an Electronics Science and Technology University (China)

Xinghui Fan, Xi'an Technological University (China)

Weiguo Liu, Xi' an Technological University (China)

Jinping Ni, Xi' an Technological University (China)

Jianhua Sun, Huanic Corporation (China)

Ailing Tian, Xi' an Technological University (China)

Fang Wang, Xi' an Institute of Applied Optics (China)

Wei Wu, Xi'an Institute of Applied Optics (China)

Renmin Yang, Xi'an Shixian Technology Company, Ltd. (China)

Shunming Yang, Xi'an Jiaotong University (China)

Xiaoxu Yang, Xi' an Institute of Optics and Precision Mechanics (China)

Xiangzhong Zeng, Mid-River Company (China)

Chunmin Zhang, Xi'an Jiaotong University (China)

Wei Zhao, Xi' an Institute of Optics and Precision Mechanics (China)

Renkui Zhou, Xi'an Institute of Optics and Precision Mechanics (China)

Changjun Zhu, Xi'an Polytechnic University (China)

\section{Program Committee}

Anand Asundi, Nanyang Technological University (Singapore)

Guangqing Du, Xi'an Jiaotong University (China)

Weiguo Liu, Xi'an Technological University (China)

Gaohong Li, Xi'an Technological University (China)

Bingcai Liu, Xi'an Technological University (China)

Jiangbo She, Xi' an Institute of Optics and Precision Mechanics (China)

Ailing Tian, Xi'an Technological University (China)

Chunhui Wang, Xi'an Technological University (China)

Hongjun Wang, Xi'an Technological University (China)

Chunmin Zhang, Xi'an Jiaotong University (China)

Xueliang Zhu, Xi'an Technological University (China) 


\section{Introduction}

Following the successes of the Annual China Western Photonics Conference in 2010, 2011, 2012 and 2013, the Shaanxi Optical Society, Shaanxi Provincial Physical Society, Optics and Photonics Society of Singapore, Xi' an Technological University, Xi' an Jiaotong University, Xi'an Institute of Optics and Precision Mechanics of CAS, Xi'an Institute of Applied Optics, and Nanyang Technological University hosted the International Conference on Photonics and Optical Engineering 2014 (icPOE 2014) 13-15 October 2014, in Xi'an, China.

The field of photonics and optical engineering is developing rapidly worldwide. Indeed, there have been significant advances in this field in China as well over the past years. There is an increasing number of photonics and optical engineering companies setting up or ramping up their manufacturing facilities here; and at the same time, some home-grown companies are show-casing innovations and technologies. In order to cater to these developments, many universities and scientific research institutions in Xi'an, Shanghai, Tianjin, and other famous cities have established the 'Photonics Research Centre'. These institutions have organized many national academic conferences for deeper academic exchanges and scientific interactions, as well as to strengthen the cultivation of the excellent talent, and finally to reach out to the younger generation and highlight them career and research options in photonics and optical engineering. The International Conference on Photonics and Optical Engineering (icPOE) is a conference of international academic exchanges about optical engineering and photonics technology. Its goal is to build a bridge between the study of photonics and optical engineering and the promotion of the application, manipulation, and measurement of new advanced technology in the context of continuous innovation and development. Leading scientists and researchers from all over the world submitted 138 papers, which are summarized under six topics:

1. Space optics

2. Spectral imaging technique

3. Photoelectric material and photoelectric devices

4. Optical design and manufacturing technology

5. Optical measurement and inspection

6. Micro-nano manufacturing and testing

The editors would like to express their thanks to all the authors who spent a lot of time and effort in the preparation of the papers. Our appreciation also goes to SPIE for providing excellent conditions for the publication of the proceeding. Our deep thanks are directed to master students Tian Yujun, Liu Ting, Bao Jianan, Li Jicheng, and Zhang Cong. The continuous help given especially by Zhou Renkui, Yang Xiaoxu and Chen Feng was the basis for making a successful icPOE 2014. 
Finally, our special thanks and appreciation goes to all friends and colleagues for sharing with us the positive result of photonics and optical engineering.

\section{Ailing Tian \\ Anand Asundi \\ Weiguo Liu \\ Chunmin Zhang}

xviii

Proc. of SPIE Vol. $9449944901-18$

Downloaded From: https://www.spiedigitallibrary.org/conference-proceedings-of-spie on 26 Apr 2023 Terms of Use: https://www.spiedigitallibrary.org/terms-of-use 